Revue musicale OICRM

\title{
La cathédrale anglicane de Québec et les organistes, 1801-1967. Adéquation ou décalage entre l'offre et la demande?
}

\section{Louis Brouillette}

Volume 2, numéro 1, 2014

URI : https://id.erudit.org/iderudit/1055853ar

DOI : https://doi.org/10.7202/1055853ar

Aller au sommaire du numéro

Éditeur(s)

OICRM

ISSN

2368-7061 (numérique)

Découvrir la revue

Citer cet article

Brouillette, L. (2014). La cathédrale anglicane de Québec et les organistes, 1801-1967. Adéquation ou décalage entre l'offre et la demande ? Revue musicale OICRM, 2(1), 212-229. https://doi.org/10.7202/1055853ar
Résumé de l'article

Cette étude analyse, selon une approche à la fois historique et socioéconomique, l'offre et la demande d'organistes à la première cathédrale anglicane construite en dehors des îles britanniques : Holy Trinity de Québec. La période étudiée est délimitée par les années 1801 et 1967, soit les années d'engagement du premier et du dix-huitième organiste de la cathédrale. Le but de cette analyse réside dans la théorisation de l'adéquation ou du décalage entre les propositions des dirigeants de la cathédrale et de la congrégation (les demandeurs) et les exigences des organistes (les offreurs). En vue de formuler des conclusions, les concepts de demande et d'offre sont expliqués dans le contexte particulier de cette étude. Les explications de ces deux termes puisent leurs sources dans la circonscription de notions qui leur sont ici intrinsèques : le terrain de recherche d'organistes, les stratégies de recrutement, le processus de sélection, les incitatifs à l'embauche, les attentes de l'employeur, le contenu des offres finales, la décision des organistes face aux offres finales, les raisons de l'arrêt de travail et la demande de pension. En plus de faire référence à certains principes de la microéconomie concernant la théorie de l'offre et de la demande, cette étude propose des éléments d'analyse propres au contexte, dont le facteur temporel relatif à la durée de recherche d'un employé. Les données qui sous-tendent l'analyse proviennent de recherches inédites dans les archives de la cathédrale Holy Trinity de Québec. Les informations colligées et analysées permettront d'ailleurs d'élargir le champ des connaissances concernant les cathédrales anglicanes en Amérique du Nord. 


\title{
La cathédrale anglicane de Québec et les organistes, 1801-1967. Adéquation ou décalage entre l'offre et la demande?
}

\author{
Louis Brouillette
}

\begin{abstract}
Résumé
Cette étude analyse, selon une approche à la fois historique et socioéconomique, l'offre et la demande d'organistes à la première cathédrale anglicane construite en dehors des îles britanniques : Holy Trinity de Québec. La période étudiée est délimitée par les années 1801 et 1967, soit les années d'engagement du premier et du dix-huitième organiste de la cathédrale. Le but de cette analyse réside dans la théorisation de l'adéquation ou du décalage entre les propositions des dirigeants de la cathédrale et de la congrégation (les demandeurs) et les exigences des organistes (les offreurs). En vue de formuler des conclusions, les concepts de demande et d'offre sont expliqués dans le contexte particulier de cette étude. Les explications de ces deux termes puisent leurs sources dans la circonscription de notions qui leur sont ici intrinsèques : le terrain de recherche d'organistes, les stratégies de recrutement, le processus de sélection, les incitatifs à l'embauche, les attentes de l'employeur, le contenu des offres finales, la décision des organistes face aux offres finales, les raisons de l'arrêt de travail et la demande de pension. En plus de faire référence à certains principes de la microéconomie concernant la théorie de l'offre et de la demande, cette étude propose des éléments d'analyse propres au contexte, dont le facteur temporel relatif à la durée de recherche d'un employé. Les données qui sous-tendent l'analyse proviennent de recherches inédites dans les archives de la cathédrale Holy Trinity de Québec. Les informations colligées et analysées permettront d'ailleurs d'élargir le champ des connaissances concernant les cathédrales anglicanes en Amérique du Nord.
\end{abstract}

Mots clés : cathédrale anglicane ; offre et demande ; organiste ; socioéconomie ; Québec.

\begin{abstract}
This article uses a historical and socioeconomic approach to analyze the supply and demand of organists at the first Anglican cathedral built outside the British Isles: Holy Trinity, in Quebec City. The period of study spans from 1801 to 1967, the tenure of the first to the seventeenth cathedral organist. The purpose of this analysis is to form a theory of the adequacy or discrepancy between the proposals of cathedral leaders and the congregation (the requesters) and the requirements of the organists (the suppliers). In order to draw conclusions, the concepts of supply and demand are explained within
\end{abstract}


the particular context of this study. The explanations of these two terms are based on the following concepts: field research carried out to find organists, recruitment strategies, the selection process, hiring incentives, employer expectations, content of final offers, the organists' decisions regarding final offers, reasons for cessation of work, and pension claims. In addition to referring to principles of microeconomics regarding the theory of supply and demand, this study provides analytical elements specific to this context, such as the time required to search for an employee. The data underlying the analysis come from original research conducted in the archives of the Cathedral of the Holy Trinity, in Quebec City. The information collected and analyzed will broaden the scope of knowledge of the Anglican cathedrals in North America.

Keywords: Anglican cathedral; organist; socioeconomics; supply and demand; Quebec.

Peu d'informations sont connues sur les organistes engagés par la première cathédrale anglicane construite en dehors des îles britanniques ${ }^{1}$, Holy Trinity de Québec $^{2}$. Néanmoins, plusieurs documents inédits des archives de la cathédrale ${ }^{3}$ permettent de connaître, notamment, les stratégies de recherche d'organistes, la provenance des musiciens, les clauses des contrats d'engagement, les causes de démission, le traitement réservé aux retraités et l'effet des gestes de reconnaissance sur la rétention des organistes.

La lecture de ces documents inédits nous pousse à se questionner sur les rapports entre les propositions des instances dirigeantes de la cathédrale (le clergé, les marguilliers, le conseil de fabrique et l'assemblée des paroissiens ${ }^{4}$ ) et les exigences des

1 L'auteur tient à remercier sincèrement James Sweeny, archiviste du diocèse anglican de Québec, pour l'accès aux sources premières.

2 Seuls quelques articles d'encyclopédies, des ouvrages généraux sur la musique au Québec et des recherches menées par des étudiants gradués donnent des informations pertinentes sur les organistes de la cathédrale Holy Trinity. Le Dictionnaire biographique du Canada décrit, par exemple, la vie d'un des 18 organistes titulaires ayant œuvré à la cathédrale Holy Trinity entre 1801 et 1967, soit John Bentley (Ryder 1983, p. 73-75) L'Encyclopédie de la musique au Canada contient pour sa part des articles sur cinq organistes de la cathédrale : John Bentley (Ryder 1993, p. 278), Henry Carter (Hayes, McGregor et Rizzo 1993a, p. 523), John Carter (Hayes, McGregor et Rizzo 1993b, p. 523), Stephen Codman (Poirier 1993, p. 672-673) et Maitland Farmer (Blakeley 1993, p. 1133). Le mémoire de maîtrise de Carole Grégoire (1990, p. 58-62, 65-67) relève avec rigueur les informations de la presse sur Bentley et Codman tandis que le mémoire de Vivianne Émond (1986, p. 53-54, 105-107) rectifie, grâce à la presse, des informations concernant les huit premiers organistes titulaires et certains remplaçants. L'ouvrage de Marie-Thérèse Lefebvre et Jean-Pierre Pinson (2009) donne quant à lui d'intéressants repères chronologiques sur Bentley, Codman et les deux Carter. La thèse de doctorat de Louis Brouillette (2009, p. 371-377) fournit également des informations biographiques sur Bentley et Codman, en plus de dresser une liste des organistes titulaires et remplaçants de 1801 à 1924. Enfin, le livre sur l'église presbytérienne St. David d'Halifax dévoile des renseignements inédits sur l'organiste Arnold Bellis (Cahill et al. 2008, p. 176-177).

3 Les archives du diocèse anglican de Québec sont conservées à l'Université Bishop's, dans l'arrondissement de Lennoxville, à Sherbrooke.

$4 \quad$ Holy Trinity possède une double identité, celle de cathédrale et d'église paroissiale. Pour cette raison, les décisions importantes sont entérinées à la réunion annuelle de l'assemblée des paroissiens (vestry). À la fondation de la cathédrale, les affaires courantes étaient réglées par le recteur (rector) et les deux marguilliers (church wardens), et à partir de 1832, c'est le conseil de fabrique (select vestry) qui s'en chargea. 
organistes. En d'autres mots, est-ce que le clergé et la congrégation de cette cathédrale anglicane sise dans une ville majoritairement francophone et catholique ont trouvé des organistes qui répondaient à leurs attentes et, en contrepartie, est-ce que les organistes engagés étaient satisfaits de leurs conditions de travail ? De plus, est-ce que les critères de sélection des organistes reflétaient réellement le profil d'organiste recherché par les ecclésiastes et la communauté ?

Il peut paraître étrange d'associer une étude sur les organistes de la cathédrale Holy Trinity à la théorie de l'offre et de la demande, qui est un modèle économique développé par la science de la microéconomie et ayant pour but de déterminer les prix du marché ${ }^{5}$. Or, cette théorie sera ici détournée de ses équations mathématiques dans le but d'offrir une perspective socioéconomique. Le présent article se présente ainsi comme une étude de vérification visant l'étayement de l'équilibre ou du déséquilibre entre l'offre et la demande d'organistes à la cathédrale Holy Trinity. La " demande » est ici associée au besoin de la cathédrale de posséder un organiste tandis que l'« offre » fait référence à la ressource productive qu'offrent les organistes, soit leur capital humain.

Les données recueillies pour cette étude ont principalement été puisées dans les archives de la cathédrale anglicane de Québec, plus particulièrement dans les procès-verbaux des réunions de l'assemblée des paroissiens et du conseil de fabrique ainsi que dans les livres de compte des marguilliers. Pour des fins de comparaison, des recherches furent également menées dans les archives d'autres églises anglicanes de Québec. La période étudiée s'étend de 1801, année de l'engagement du premier organiste de la cathédrale Holy Trinity, à 1967, année de l'entrée en fonction du dix-huitième organiste titulaire.

Cette présente étude, qui est basée sur l'analyse de données recueillies dans les archives, s'avère primordiale pour la connaissance de la vie musicale non seulement de la cathédrale Holy Trinity, mais également de la ville de Québec et de la communauté anglicane nord-américaine, voire occidentale. La littérature - peu abondante - sur le travail des organistes de Holy Trinity (voir note 2) se basait, jusqu'à présent, presque uniquement sur les faits rapportés par la presse. L'absence d'inventaire des archives de la cathédrale anglicane de Québec avait d'ailleurs rebuté Vivianne Émond à y effectuer des recherches archivistiques (Émond 1986, p. 106) et Carole Grégoire avait souligné dans son mémoire le rôle-clé que jouerait les recherches dans les archives pour les études subséquentes sur les orgues et les organistes du Québec (Grégoire 1990, p. 72).

5 La confrontation de l'offre et de la demande, en parfaite concurrence, établit en fait un prix d'équilibre (voir Defalvard 2003, p. 25). 


\begin{tabular}{|c|c|c|c|}
\hline Organistes & $\begin{array}{l}\text { Période } \\
\text { d'emploi } \\
\text { comme } \\
\text { organiste à } \\
\text { Holy Trinity }\end{array}$ & $\begin{array}{l}\text { Lieu de résidence } \\
\text { ou de travail } \\
\text { avant } \\
\text { l'engagement à } \\
\text { Holy Trinity }\end{array}$ & $\begin{array}{l}\text { Motif de I'arrêt de travail à } \\
\text { Holy Trinity }\end{array}$ \\
\hline John Bentley & $1801-1813$ & Québec & Décès \\
\hline Stephen Codman & $1816-1852$ & Angleterre & Décès \\
\hline John Carter & $1853-1856$ & Londres, Angleterre & $\begin{array}{l}\text { Démission (engagement à la } \\
\text { cathédrale St. James, Toronto) }\end{array}$ \\
\hline Henry Carter & $1857-1861$ & Angleterre & Démission/congédiement \\
\hline James Pearce & $1862-1865$ & Alnwick, Angleterre & Démission \\
\hline $\begin{array}{l}\text { Frederick William } \\
\text { Mills }\end{array}$ & $1865-1872$ & Angleterre? & $\begin{array}{l}\text { Démission (engagement à l'église } \\
\text { Christ Church, Ottawa) }\end{array}$ \\
\hline $\begin{array}{l}\text { John Watson } \\
\text { Warman }\end{array}$ & $1872-1873$ & Angleterre & Non renouvellement du contrat \\
\hline Henry Bradley & $1873-1874$ & Angleterre & Démission \\
\hline $\begin{array}{l}\text { Edward Arthur } \\
\text { Bishop }\end{array}$ & $1874-1924$ & Angleterre & Retraite \\
\hline G. H. Harvey & $1924-1929$ & $\begin{array}{l}\text { Église St. } \\
\text { Matthew's, Québec }\end{array}$ & Démission \\
\hline Maitland Farmer & $1929-1932$ & Angleterre & $\begin{array}{l}\text { Démission (engagement à l'église } \\
\text { St. Paul's, Toronto) }\end{array}$ \\
\hline William Rains & $1932-1936$ & $\begin{array}{l}\text { Cathédrale de } \\
\text { Ripon, Angleterre }\end{array}$ & Démission (retour en Angleterre) \\
\hline Sydney Martin & $\begin{array}{l}\text { 1936-[au } \\
\text { moins } \\
\text { jusqu'en } \\
1942]\end{array}$ & Québec & Démission \\
\hline John Clarke & $?-1949$ & $?$ & Démission \\
\hline Arnold Bellis & 1950 & $\begin{array}{l}\text { Église } \\
\text { presbytérienne St. } \\
\text { David, Halifax }\end{array}$ & Démission \\
\hline George Chubb & $1951-1954$ & $?$ & Démission \\
\hline Richard Carroll & $1954-1967$ & $?$ & Démission \\
\hline Stephen Crisp & $1967-1970$ & $\begin{array}{l}\text { Cathédrale de } \\
\text { Cantorbéry, } \\
\text { Angleterre }\end{array}$ & $?$ \\
\hline
\end{tabular}

Figure 1: Les organistes titulaires de la cathédrale Holy Trinity de Québec de 1801 à 1970.

LA NOTION DE DEMANDE : LE RECRUTEMENT ET L'ENGAGEMENT SELON LA PERSPECTIVE DE L'EMPLOYEUR

La musique joue un rôle viscéral dans le culte anglican et surtout dans ses cathédrales, qui ont impérativement besoin d'un organiste pour préparer et présenter 
la portion musicale des offices ${ }^{6}$. C'est pourquoi de 1801 à 1967 , à chaque fois qu'un organiste de la cathédrale Holy Trinity de Québec démissionnait, une demande de remplacement se créait. Sans être soutenus par un processus réflexif nécessairement conscient, des actions étaient menées : 1) délimiter un terrain de recherche d'organistes ; 2) déterminer des stratégies de recrutement ; 3) définir un processus de sélection ; 4) élaborer des incitatifs à l'embauche ; 5) clarifier les attentes des dirigeants de la cathédrale ; et 6) rédiger une offre finale. Toutes ces phases de recrutement et d'engagement concourraient à satisfaire une demande, c'est-à-dire de bénéficier du capital humain d'un organiste. La caractérisation de ces phases permettra ultérieurement de comparer l'offre et la demande dans le but d'y discerner une adéquation ou un décalage.

Pour mettre en contexte les organistes qui sont cités dans cette étude, la figure 1 propose une liste inédite des 18 premiers organistes titulaires de la cathédrale Holy Trinity en mentionnant notamment le lieu de résidence ou de travail de ces musiciens avant d'assumer leur poste à Québec et le motif de leur arrêt de travail à Holy Trinity.

\section{Le terrain de recherche}

Étant donné le nombre limité d'organistes à Québec au XIX siècle et vu les relations privilégiées de plusieurs membres de la congrégation avec leur mère patrie, il semblait évident, à l'époque, que le recrutement devait s'effectuer parmi les organistes d'Angleterre $^{7}$. À la suite du décès de Stephen Codman, les marguilliers suggérèrent justement au conseil de fabrique de se " procurer » un organiste en Angleterre (CAQ, PV CF 22 octobre $1852^{8}$ ). De même, après la démission de Frederick William Mills, les lettres annonçant l'ouverture du poste d'organiste à la cathédrale furent envoyées uniquement à des Anglais (CAQ, PV CF 7 septembre 1872).

En 1883, le territoire de recrutement changea radicalement. En fait, suite à la première démission d'Edward Arthur Bishop ${ }^{9}$, la publicité au sujet du poste

6 Dans la tradition anglicane, l'organiste assure généralement la tâche de maître de chapelle. À la cathédrale anglicane de Québec, tous les organistes titulaires engagés entre 1801 et 1967 dirigeaient la ou les chorale(s) en plus de jouer de l'orgue. L'organiste Stephen Codman arrêta toutefois ses activités de chef de chœur en 1844, année de la dissolution de la maîtrise vêtue de surplis. Les procès-verbaux des assemblées de paroissiens de 1845 à 1850 montrent que c'est le musicien amateur Archibald Campbell qui assura la direction du nouveau chœur bénévole. Campbell a probablement assumé la fonction de chef de chœur jusqu'au décès de Codman, en 1852.

7 Le premier organiste de la cathédrale Holy Trinity, l'Anglais John Bentley, résidait toutefois à Québec avant son engagement comme organiste en 1801. Employé de la fonction publique à Québec à partir de 1791, musicien de théâtre et professeur de musique, il assuma également la fonction d'organiste à la cathédrale catholique de Québec (Brouillette 2009, p. 373).

8 La présente note bibliographique fait référence à un document du fonds Cathédrale de Québec (CAQ), soit un procès-verbal (PV) de la réunion du conseil de fabrique ( $\mathrm{CF}$ ) du 22 octobre 1852. Une liste de toutes les abréviations employées dans cet article précède la bibliographie.

9 Bishop resta finalement à l'emploi de la cathédrale Holy Trinity jusqu'en 1924. Cette année-là, il donna sa démission après 50 ans de travail comme organiste titulaire. 
d'organiste s'effectua principalement en Amérique du Nord ; les deux candidats privilégiés résidaient à Ottawa et à Philadelphie (CAQ, PV CF 23 février 1883). En 1841, une première démarche de recrutement avait néanmoins été menée aux États-Unis, plus particulièrement à Boston (CAQ, PV CF 8 juin 1861), en plus des traditionnelles communications par écrit envoyées en Angleterre par le recteur et les marguilliers (CAQ, PV CF 13 avril 1861).

En 1949, la recherche d'organiste s'est opérée uniquement au Canada. En fait, le recteur a écrit au Collège canadien des organistes, au Conservatoire de musique de Toronto et à l'organiste de la cathédrale St. Jame's de Toronto (CAQ, PV CF 21 octobre 1949). C'est Arnold Bellis, un Anglais excentrique émigré en 1947 travaillant depuis 18 mois à l'église presbytérienne St. David d'Halifax (Cahill et al 2008, 176), qui fut choisi comme titulaire.

Cette recherche d'organiste menée exclusivement au Canada semble toutefois être exceptionnelle. En effet, en 1967, à la suite de la démission de Richard Carroll, le recrutement s'effectua tant au Canada qu'en Angleterre (CAQ, PV CF 23 mai 1967). C'est encore un Anglais qui obtint le poste, soit Stephen Crisp, qui était l'organiste assistant à la cathédrale de Cantorbéry.

Bien que le territoire de recherche se soit modifié au cours de la période étudiée, le profil des candidats retenus est resté - à quelques exceptions près ${ }^{10}$ - le même tout au long de cette période, soit des organistes masculins professionnels formés en Angleterre.

Les stratégies de recrutement

Alors que le territoire de recherche s'est transformé au fil des années, certaines stratégies de recrutement ont constamment été utilisées, comme le recours aux références, et d'autres, comme la publicité dans les journaux, ont été employées quelques années seulement. En fait, les archives de la cathédrale mentionnent que des annonces d'ouverture du poste d'organiste ont été acheminées dans les journaux seulement en 1861 et 1949 (CAQ, PV CF 8 juin 1861 et 21 octobre 1949).

En revanche, l'emploi à des références est rapporté dans plusieurs procès-verbaux. Le plus souvent, les dirigeants de Holy Trinity demandaient à des organistes engagés par d'importantes églises anglicanes - de Londres, Toronto, Victoria (ColombieBritannique) et Kingston (Ontario) - de leur fournir des suggestions. Le londonien Henry May a d'ailleurs référé Henry Bradley et Edward Arthur Bishop, qui ont successivement été engagés à la cathédrale Holy Trinity (CAQ, PV CF 29 juillet 1873 et 5 septembre 1874). Les recommandations du Collège canadien des organistes, en 1949, et des directeurs d'écoles supérieures de musique - le Conservatoire de musique de Toronto en 1949 et le Royal School of Church Music d'Angleterre en 1967 - ont également été sollicitées (CAQ, PV CF 21 octobre 1949 et 23 mai 1967).

10 Aucune information n'a été retracée au sujet de l'endroit où ont été formés G. H. Harvey et Sydney Martin, qui ont tous deux travaillé comme organistes à la cathédrale Holy Trinity et à l'église anglicane St. Matthew de Québec. Quant à George Chubb, il a reçu des leçons de musique de son père en ColombieBritannique (Waterlow 1993, p. 642), mais on ne sait pas s'il est allé parfaire sa formation en Angleterre. 
Deux organistes démissionnaires de la cathédrale Holy Trinity ont aussi soumis des noms de successeurs. En 1865, James Pearce proposa les services de son frère qui résidait en Angleterre (CAQ, PV CF 24 janvier 1865), mais les dirigeants de la cathédrale ont préféré engager Frederick William Mills. Lorsque Mills démissionna à son tour en 1873, il offrit au conseil de fabrique de rechercher un successeur durant ses vacances en Angleterre. À son retour, trois mois plus tard, il donna une liste d'organistes dans laquelle se trouvait le nom de John Watson Warman, qui succéda à Mills comme organiste titulaire (CAQ, Annual Report of the Church Wardens of the Cathedral, Quebec 1873, p. 6). Entre 1801 et 1967, Warman fut le seul organiste engagé par la cathédrale à avoir été référé par un autre organiste de Holy Trinity et il aussi le seul dont le premier contrat d'un an n'a pas été renouvelé par le conseil de fabrique.

\section{Le processus de sélection}

Le processus de sélection des organistes de la cathédrale Holy Trinity s'est modifié au cours des ans en s'adaptant au déplacement du territoire de recherche. À l'époque durant laquelle le recrutement s'effectuait seulement en Angleterre, les dirigeants de la cathédrale se fiaient principalement, et parfois uniquement, aux recommandations écrites des organistes anglais. Lorsque le recrutement commença à s'effectuer davantage en Amérique du Nord, il fut alors possible de demander aux candidats de passer une audition ou une entrevue à Québec. Selon les archives de la cathédrale, C. A. Harriss d'Ottawa serait le premier organiste à avoir passé une audition ; c'était en 1883. L'orgue Warren de la cathédrale - qui avait été installé l'année précédente - était toutefois en si mauvais état que le conseil de fabrique, en réponse au mécontentement de Harriss, a accepté que le candidat soit convoqué à une seconde audition lorsque l'orgue serait réparé (CAQ, PV CF 9 mars 1883).

Les comités de sélection étaient généralement formés d'un marguillier et de membres de la congrégation qui connaissaient la musique. En 1883, par exemple, les musiciens amateurs William Darling Campbel1 ${ }^{11}$ et James A. Sewell ${ }^{12}$, en collaboration avec un marguillier, avaient la responsabilité de s'assurer des qualifications des candidats (CAQ, PV CF 23 février 1883).

Il ne semble pas, néanmoins, que tous les candidats, à partir de 1883, aient été auditionnés. Les procès-verbaux du conseil de fabrique mentionnent à plusieurs reprises que des interviews ont été menées, mais la signification du verbe anglais " to interview " est ambigüe : est-ce que ce terme fait référence uniquement à une entrevue ou suppose-t-il implicitement une audition? Quoi qu'il en soit, il n'était pas exigé des organistes qui habitaient en Angleterre au $x^{e}$ siècle de se rendre à Québec

11 Campbell était un violoncelliste amateur qui faisait partie du chœur bénévole de la cathédrale. Les archives de la cathédrale conservent d'ailleurs un manuscrit de théorie musicale et de solfège lui ayant appartenu (Brouillette 2009, p. 14-15).

12 Sewell assura temporairement la tâche d'organiste après le décès de Stephen Codman en 1852 (CAQ, CF 27 décembre 1852) et la démission de Frederick William Mills à l'été 1872 (CAQ, AP 14 avril 1873). Il avait également été organiste pendant plusieurs années à l'église Trinity, fondée en 1825 par son père, l'honorable Jonathan Sewell (ETC, Centenary of Trinity Church, Quebec, 1825-1925 1925, p. 6-7). 
pour le processus de sélection. En 1967, par exemple, un dénommé Ronald Blaire a « interviewé » en Angleterre les deux candidats anglais (CAQ, PV CF 23 mai 1967).

Les incitatifs à l'embauche

Dans le but d'attirer les meilleurs organistes, les dirigeants de la cathédrale Holy Trinity se sont souvent servis de deux mesures incitatives : l'indemnité de déménagement et la hausse salariale à l'embauche. À l'époque de la recherche d'organiste exclusivement en Angleterre, un montant prédéterminé était destiné au passage outre-Atlantique. En 1852, la cathédrale offrait un généreux montant maximal de 25 livres sterling à l'organiste qui remplacerait Stephen Codman (CAQ, PV CF 8 novembre 1852). L'indemnité fut diminuée à seulement 15 livres sterling en 1861 (CAQ, PV CF 31 juillet 1861). Le conseil de fabrique offrait ce même montant onze ans plus tard (CAQ, PV CF 7 septembre 1872), puis en 1874, la somme réservée au déménagement de l'Angleterre à Québec fut augmentée à 20 livres sterling (CAQ, PV CF 12 octobre 1874).

La cathédrale Holy Trinity ne fut pas la seule église anglicane de Québec à payer le déménagement d'organistes anglais. En 1880, l'église St. Matthew's a défrayé le transport de l'Angleterre à Québec de son nouvel organiste (ESM, Church Wardens' Report. St. Matthew's Church 1880, p. 4).

$\mathrm{Au} \mathrm{Xx}^{\mathrm{e}}$ siècle, l'indemnité offerte par la cathédrale variait selon la provenance de l'organiste et de ses demandes. Ainsi, en 1950, le conseil de fabrique consentit à donner 100 dollars à Arnold Bellis pour son déménagement (CAQ, PV CF 27 février 1950). Un an plus tard, George Chubb reçut plus du triple du montant, soit 330 dollars (CAQ, PV CF 15 mars 1951). Cette différence significative s'explique par le lieu de résidence des deux organistes avant qu'ils ne soient employés à Holy Trinity : Bellis habitait à Halifax tandis que Chubb devait résider beaucoup plus loin, son père était d'ailleurs organiste à l'église St. John de Victoria (Waterlow 1993, p. 642).

Comme l'indemnité de déménagement au $\mathrm{xx}^{\mathrm{e}}$ siècle, la hausse salariale à l'embauche fut une mesure incitative qui fut offerte de façon occasionnelle. Dans le but d'attirer des organistes compétents, voire les meilleurs organistes, les dirigeants de la paroisse ont parfois augmenté le salaire avant de publiciser l'offre d'emploi. Après le décès de Stephen Codman, par exemple, le salaire de 1'organiste doubla, passant de 50 à 100 livres sterling (CAQ, PV CF 8 novembre 1852).

À l'assemblée annuelle des paroissiens de 1873, à la suite du non renouvellement du contrat de John Watson Warman, c'est William Darling Campbell qui suggéra une hausse de salaire en vue de trouver le " meilleur talent ${ }^{13}$ " (CAQ, PV AP 14 avril 1873). À l'assemblée paroissiale de l'année suivante, c'est encore Campbell qui proposa un salaire de 600 dollars pour le futur organiste au lieu des 400 dollars jusque-là alloués. (CAQ, PV AP 6 avril 1874). Près d'un siècle plus tard, soit à la suite de la démission de Richard Carroll en 1967, le conseil de fabrique autorisa à hausser, si nécessaire, le 
salaire à 4000 dollars dans le but d'attirer un organiste dont « la stature serait proportionnelle aux exigences de la cathédrale Holy Trinity ${ }^{14}{ }$ (CAQ, PV CF 23 mai 1967).

Les attentes de l'employeur

Le manque de réflexion concernant le profil désiré d'organiste a parfois suscité des mécontentements auprès des paroissiens et des dirigeants de la cathédrale après l'engagement de l'organiste. La sélection d'œuvres non coutumières et la présentation de nombreux concerts par Maitland Farmer a, par exemple, dérouté plusieurs membres du conseil de fabrique qui avaient pourtant entériné sa nomination en 1929. Après la démission de Farmer en 1932, les marguillers ont demandé au conseil de fabrique de préciser le type d'organiste dont la cathédrale désirait engager : " celui dont la spécialité est le concert [en référence à Farmer], celui qui privilégie un chœur se consacrant à un répertoire particulier (dont les solo anthems) ou celui qui dirige un chœur dans le but d'encourager et de supporter le chant de l'assemblée ${ }^{15}$ " (CAQ, PV CF 27 janvier 1932). À l'unanimité, les membres du conseil de fabrique ont exigé que le choix de l'organiste soit principalement basé sur la capacité du candidat à former une chorale qui inciterait les paroissiens à chanter avec cœur ${ }^{16}$.

$\mathrm{Au} \mathrm{XIX}^{\mathrm{e}}$ siècle, il semble que les recruteurs anglais n'aient pas reçu d'instructions précises pour les critères de sélection, hormis le fait que les organistes devaient être compétents, voire les meilleurs talents disponibles. Au Xx ${ }^{\mathrm{e}}$ siècle, les postulants furent de plus en plus nombreux et le conseil de fabrique ajouta parfois des critères de sélection extramusicaux, dont l'âge des candidats. En 1967, par exemple, suite à la recommandation de George May, organiste à l'église St. George de Kingston, seuls les deux plus jeunes des trois postulants furent rencontrés (CAQ, PV CF 23 mai 1967).

\section{Les offres finales}

Les archives de la cathédrale anglicane de Québec conservent peu d'informations sur les clauses des contrats des organistes. C'est en parcourant les procèsverbaux du conseil de fabrique et de l'assemblée des paroissiens qu'il devient possible de concevoir la tâche globale des organistes engagés à la cathédrale, c'est-à-dire le nombre d'offices durant lesquels ils devaient être présents et le nombre de répétitions qu'ils devaient diriger en tant que maître de chapelle. Durant la période à l'étude, la tâche de l'organiste semble avoir peu changée, sauf en ce qui concerne les répétitions

14 "[...] an organist of the stature commensurate with the requirments [sic] of the Cathedral of the Holy Trinity".

15 "The wardens then request the meeting to express its view as to what qualifications in an organist were to be considered as the most needed and desired, in making the choice of an organist for this parish; it was instanced that the specialty of one organist might be recitals, of another the training of a choir in a specialized way (special anthems solos \&c) of another to train the choir with the chief view that it should give leadership and encouragement in order to promote congregational singing. "

16 À l'époque de Farmer, la cathédrale comptait déjà deux chœurs : une chorale bénévole mixte qui chantait les deux offices du dimanche et une maîtrise de jeunes garçons qui répétaient trois fois par semaine (CAQ, Church Wardens' and Other Annual Reports. Cathedral of the Holy Trinity, Quebec. [For the year] 1930 1931, p. 39). 
de chœur, dépendamment de la présence ou l'absence de la maîtrise de jeunes garçons ${ }^{17}$.

Les archives de la cathédrale conservent toutefois une copie du contrat d'engagement de James Pearce qui a été signé le 8 novembre 1861 :

Madame Noble est autorisée à engager monsieur James Pearce comme organiste de la cathédrale [anglicane] de Québec pour un an au salaire annuel de 350 dollars. Les tâches sont les suivantes : faire répéter le chœur au moins une fois par semaine ; jouer de l'orgue et diriger la portion musicale des offices dominicaux du matin et de l'après-midi, de deux offices de l'après-midi par semaine durant le Carême, des offices du matin de la semaine sainte et des deux jours suivants Pâques et la Pentecôte, de l'office du matin des principales fêtes et des offices du matin et de l'après-midi de Noël et du Vendredi saint ${ }^{18}$ (CAQ, PV CF $1^{\text {er }}$ mars 1862).

Ce contrat montre de façon représentative les offices durant lesquels tous les organistes titulaires de la cathédrale anglicane de Québec devaient être présents.

LA NOTION D'OFFRE : L'ENGAGEMENT ET L'ARRÊT DE TRAVAIL SELON LA PERSPECTIVE DE L'EMPLOYÉ

Le pouvoir des organistes dans le processus de l'offre et de la demande - c'est-à-dire entre le besoin de la cathédrale de posséder un organiste et l'offre de service des organistes - s'est entre autres réalisé au moment de l'engagement. C'est à cette étape que les musiciens pouvaient négocier leurs conditions de travail et lorsque la concurrence était faible, les candidats bénéficiaient d'un pouvoir de négociation plus élevé.

Certains interprètes ont tout simplement refusé de signer un contrat, ce qui indique un décalage entre l'offre et la demande, c'est-à-dire entre les critères d'engagement du demandeur de service (la cathédrale) et les exigences de l'offreur (l'organiste). Ce décalage s'est également créé lorsque les organistes engagés à la cathédrale décidaient de mettre un terme à leur contrat. Étant donné que leur emploi ne correspondait plus à leurs attentes, ils décidaient d'arrêter de travailler à la cathédrale. Les raisons qui les ont poussés à cette décision sont variées et parfois extérieures aux conditions de travail.

La décision des organistes face aux offres finales d'engagement

Peu d'organistes engagés par la cathédrale Holy Trinity ont négocié leur contrat de travail. Ils ont généralement accepté les conditions de leur futur employeur, et cela

17 La maîtrise pouvait avoir des répétitions régulières jusqu'à trois fois par semaine.

18 "Mrs Noble is authorised to engage Mr. Ja[me]s Pierce [sic] as organist of the Cathedral Quebec for one year at a salary of 350 \$ per annum. The duties being the following: to rehearse the choir at least once every week; to perform on the organ [and] conduct the musical services on the Sundays, morning [and] afternoon services, two afternoon services weekly during Lent, daily morning service in passion week [and] in the two days following Easter [and] Whitsunday, morning service on the principal festivals, and morning [and] afternoon services on Christmas [and] Good Friday. " 
sans modification. Un dénommé Mills semble toutefois avoir négocié son salaire. À l'assemblée des paroissiens de 1874, il avait été convenu d'augmenter le traitement du futur organiste à 600 dollars (CAQ, PV AP, 6 avril 1874). 19 jours plus tard, le conseil de fabrique approuvait l'engagement de Mills avec un salaire ne dépassant pas 800 dollars ; les membres du conseil se sont engagés à effectuer une quête spéciale durant l'année pour payer les 200 dollars de différence. Pour une raison inconnue, Mills n'a finalement pas occupé le poste d'organiste à la cathédrale et le nouvel organiste, Edward Arthur Bishop, a été engagé avec le salaire négocié par Mills (CAQ, PV AS 17 avril 1876).

Peu d'informations ont été révélées sur les raisons pour lesquelles certains organistes n'ont pas accepté le poste à Holy Trinity après avoir reçu une offre finale. On ne connaît pas, notamment, les causes du refus des dénommés Hitchens en 1861, Dickinson en 1874 et Bancroft de Winnipeg en 1932. Toutefois, on sait que Penn Spicer, qui avait été engagé en janvier 1932, a reçu, avant son entrée en poste prévue en mars, une contre-offre de son ancien employeur pour le retenir à Yarmouth, en NouvelleÉcosse. Spicer a décidé de résilier son engagement à la cathédrale Holy Trinity pour accepter la contre-offre avantageuse de l'église Holy Trinity de Yarmouth (CAQ, PV CF 17 janvier 1932).

\section{Les causes de l'arrêt de travail}

Pour les organistes qui sont demeurés en poste au moins quelques mois (ceux qui se trouvent dans la figure 1), cinq causes principales sont à l'origine de leur arrêt de travail à la cathédrale Holy Trinity : 1) le non renouvellement de leur contrat (ou le licenciement), 2) l'acceptation d'un poste d'organiste encore plus prestigieux, 3) le désir de retourner en Angleterre, 4) la vieillesse, et 5) la mort. Il est également possible de supposer des causes de démission qui ne sont pas mentionnées dans les documents d'archives. La condition de l'orgue, par exemple, a probablement influencé la décision prise en décembre 1882 par Edward Arthur Bishop sur son avenir à la cathédrale. Bishop était sûrement déçu du nouvel orgue Warren installé quelques mois avant sa démission. En fait, cet orgue, attendu depuis neuf ans ${ }^{19}$, a été livré en 1882 avec plusieurs défauts (CAQ, PV CF 23 février 1883).

Parmi toutes les causes susmentionnées d'arrêt de travail, la plus noble demeure la " promotion ", soit l'acceptation d'un poste d'organiste dans une église plus importante. En 1856, John Carter démissionna de Holy Trinity pour devenir l'organiste de l'imposante cathédrale St. James de Toronto (Hayes, McGregor et Rizzo 1993b, p. 523), dont la construction s'était terminée trois ans auparavant. En 1932, ce fut au tour de Maitland Farmer de quitter Québec pour occuper le poste d'organiste d'une autre église anglicane de Toronto, St. Paul's. Dans le rapport annuel

19 En 1873, le conseil de fabrique a demandé au facteur Samuel Russell Warren une soumission pour la construction d'un orgue, en remplacement du Bevington installé en 1847 (CAQ, PV CF 16 juillet 1873). Un an plus tard, le conseil de fabrique approuvait une résolution sur la nécessité de se procurer un nouvel orgue (CAQ, PV CF 12 juillet 1874). 
de 1931, les marguilliers et le recteur félicitèrent Farmer pour sa promotion ; selon les marguilliers, St. Paul's possédait à cette époque le plus important chœur au Canada (CAQ, Church Wardens' and Other Annual Reports. Cathedral of the Holy Trinity, Quebec [For the year] $19311932^{20}$, p. 8 et 43).

La raison du départ de l'Anglais William Rains en 1936 fut tout aussi compréhensible pour les paroissiens de Holy Trinity. Après quatre ans de travail à la cathédrale de Québec, Rains souhaitait rejoindre sa famille en Angleterre (CAQ, PV CF 8 mai 1936). Il se trouva un poste à l'église historique de Northallerton, dans le Yorshire (CAQ, Church Wardens' and Other Annual Reports. Cathedral of the Holy Trinity, Quebec. [For the year] 1936 1937, p. 36). Avant son séjour à Québec, Rains était organiste assistant à la cathédrale de Ripon, également dans le Yorkshire (Church Wardens' and Other Annual Reports. Cathedral of the Holy Trinity, Quebec. [For the year] 1932 1933, p. 10).

Pour les deux premiers organistes de Holy Trinity, leur départ fut indépendant de leur volonté, car ils sont tous deux décédés alors qu'ils étaient encore à l'emploi de la cathédrale. John Bentley a trépassé le 10 novembre 1813 tandis que son successeur, Stephen Codman, a succombé à une maladie le 6 octobre 1852, après 36 ans de service à la cathédrale.

\section{La demande de pension : l'affaire Bishop}

Un seul organiste de la cathédrale anglicane a démissionné de son poste à cause de son âge avancé. Dans sa lettre du 2 mars 1923 adressée au recteur Richmond Shreve, l'organiste Edward Arthur Bishop explique qu'il :

Y a un louable, voire enthousiaste, désir d'améliorer la chorale par l'ajout massif de membres, ce qui rendrait la portion musicale des offices digne des exigences des cathédrales. [...] Pour y arriver, il faut l'énergie, le courage et la persévérance qu'on associe à la jeunesse ou au début de la vie ${ }^{21}$ (CAQ, PV CF 13 mars 1923).

Bishop demande ensuite de recevoir une pension. Il écrit :

Pendant plus de 48 ans, j'ai servi la congrégation de la cathédrale avec un salaire qui, spécialement ces dernières années, était plus bas que ceux offerts ailleurs au Canada avec des conditions similaires. De toute façon, les économies pour les vieux jours de loisir ou d'oisiveté n'ont pu être effectuées et le salaire que je reçois actuellement est à peine équivalent aux dépenses engendrées par une vie ordinaire. Quelques-uns de mes amis m'ont dit qu'il serait possible de recevoir un revenu approprié de retraite si je le demandais, mais je ne suis pas sûr qu'on réalise qu'un pourcentage ordinaire d'un important salaire engendre

20 Ces rapports annuels de l'année finissant le 31 décembre 1931 ont été imprimés en janvier 1932. En fait, depuis 1920, la réunion annuelle des paroissiens - lors de laquelle sont lus les rapports - se déroule le troisième lundi de janvier au lieu du lundi suivant Pâques ; les rapports couvrant l'année se terminant le 31 décembre sont donc imprimés, depuis ce temps, au mois de janvier suivant.

21 "There is a commendable, even an enthusiastic desire to improve the choir by adding largely to its numbers and thus making the musical services generally such as the dignity of a cathedral church demands. [...] A forward movement needs that activity, courage and perseverance that one associates with youth or early life. " 
une pension raisonnable tandis que ce même pourcentage sur un petit salaire est vraiment inadéquat ${ }^{22}$ (CAQ, PV CF 13 mars 1923).

Même si Bishop fut le premier employé de la cathédrale anglicane de Québec à solliciter un revenu de retraite, le conseil de fabrique ne refusa pas sa demande (CAQ, PV CF 13 mars 1923). Alors que Bishop recevait un salaire de 1650 dollars en tant qu'organiste, une pension annuelle de 1500 dollars lui fut accordée à partir de novembre 1824 (CAQ, Livre de comptes des marguilliers 1911-1926, p. 98). Son revenu de retraite était probablement aussi élevé qu'il le désirait et, comme le démontre la figure 2, il était même identique au salaire du nouvel organiste, G. H. Harvey ${ }^{23}$. Le salaire de l'organiste titulaire devint supérieur à la pension de Bishop seulement en 1929, avec l'engagement de Maitland Farmer, qui succéda à Harvey.

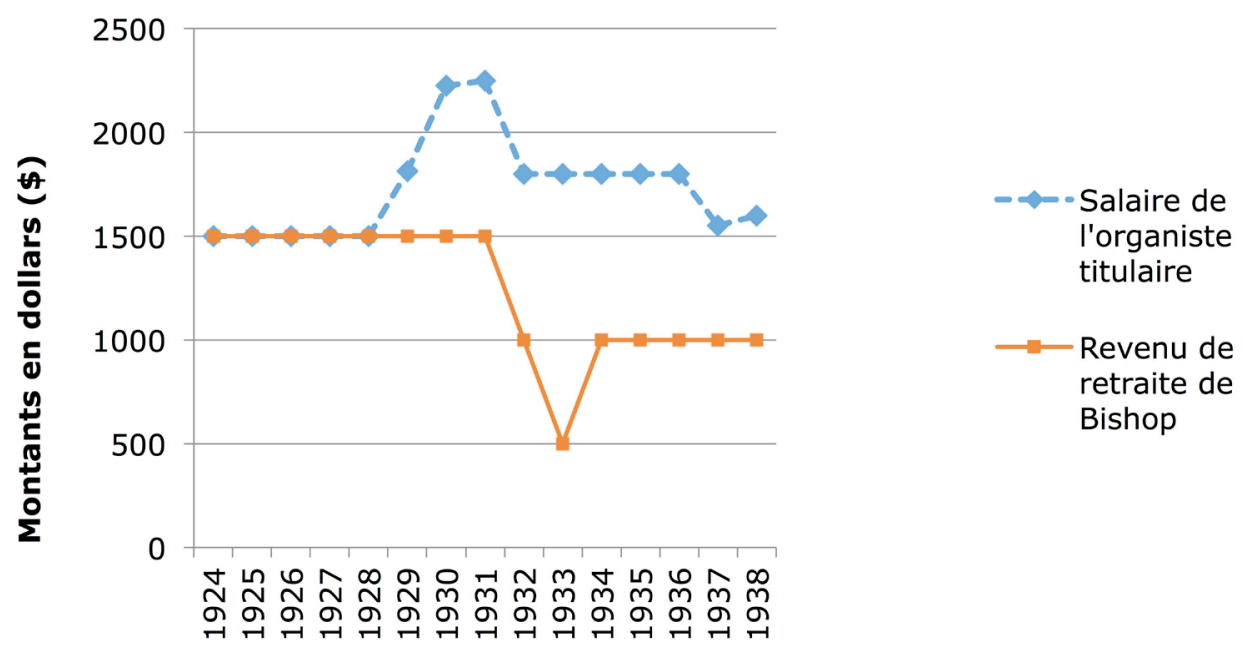

Années

Figure 2: Le salaire de l'organiste titulaire de la cathédrale anglicane de Québec de 1824 à 1838 vs le revenu de retraite d'Edward Arthur Bishop.

Les frais de la pension de Bishop étaient divisés, à partir de 1924, en trois parts égales. La cathédrale payait annuellement 500 dollars tandis que les deux autres montants de 500 dollars provenaient de deux riches paroissiens (CAQ, PV CF 27 janvier 1932) : Vesey Boswell, un des plus importants brasseurs de Québec, et William Price,

22 "For over 48 years I have served the Cathedral Congregation at a salary that was, especially in later years, below the scale prevailing elsewhere in Canada under similar conditions. At any rate, provision for an old age of leisure or idleness could not be made, and even now the salary I receive is barely equal to the demands of plain living. It has been intimated to me by some of my friends that there would be no difficulty in providing a suitable pension should I wish to retire, but I am not sure that it was realized that while an ordinary percentage of a large salary produces a reasonable pension the same percentage on a small salary is quite inadequate. "

23 Bien que le salaire de l'organiste ait diminué de 1650 à 1500 dollars à l'engagement de Harvey, ce montant représente environ le double de ce qu'il gagnait à l'église St. Matthew de Québec. En 1916, son salaire à St. Matthew s'élevait seulement à 700 dollars (ESM, Annual Reports. St. Matthew's Church, Quebec 1916, p. 10) alors que Bishop était payé 1400 dollars à la cathédrale (CAQ, Annual Reports. Cathedral of the Holy Trinity, Quebec 1916, p. 11). 
un prospère industriel, propriétaire de la Price Brothers and Compagny qui était spécialisée dans la coupe du bois, le sciage et la fabrication de papier.

À partir de 1932, les revenus de retraite de Bishop diminuèrent pendant deux ans et l'ancien organiste a failli ne plus recevoir de pension. À cette époque, beaucoup de paroissiens furent durement touchés par la "récession économique aigüe ${ }^{24}$ " et les finances de la cathédrale se dirigeaient vers un important déficit (CAQ, Lettre des marguilliers F. M. Gibaut et G. F. Gibsone aux membres de la congrégation de la cathédrale Holy Trinity, 20 septembre 1932). En 1932, la Price Brothers and Compagny devint insolvable et par conséquent, la succession de Price ne pouvait plus cotiser à la pension, c'est pourquoi Bishop a seulement reçu 1000 dollars de revenus de retraite cette année-là.

L'année suivante, ce fut au tour de la cathédrale de retirer sa contribution, les marguilliers ayant recommandé de cesser les cotisations à la pension de Bishop en vue de diminuer le déficit (CAQ, Lettre des marguilliers F. M. Gibaut et G. F. Gibsone à un dénommé Graham, 13 octobre 1932). Bishop reçut en 1933 que les 500 dollars donnés par Boswell. Puis au début de l'année 1934, Boswell a annoncé que ses finances ne lui permettaient plus de défrayer la pension de Bishop (CAQ, PV CF 7 février 1934). Le conseil de fabrique décida de cotiser à nouveau à la pension de son ancien organiste, cette fois-ci à raison de 1000 dollars par année (CAQ, PV CF 15 février 1934). Bishop a reçu cette rente jusqu'à son trépas en 1938.

Bien que l'octroi d'une pension à un organiste demeure un fait rare dans le monde, le cas Bishop n'est pas unique. Ruth Handley, organiste de l'église anglicane Trinity de Québec à partir de 1903, a également reçu des revenus de retraite à la même époque que Bishop, plus précisément vers la fin de l'année 1936, alors qu'elle avait accumulé 33 ans de service (ETC, Trinity Church, Québec. Financial Statements [for the] Year Ending December 31st, 1936 1937). Sa pension ne l'a cependant pas empêché d'occuper le poste d'organiste à St. Peter de Limoilou à partir de mars 1937 (ESP, Livre de comptes de l'église St. Peter 1930-1937). Son salaire annuel de 135 dollars de St. Peter s'additionnait ainsi à sa rente de 250 dollars de l'église Trinity. Handley a travaillé à St. Peter au moins jusqu'en 1948 (ESP, Livre de comptes de l'église St. Peter 1938-1948).

À la cathédrale de Hereford, en Angleterre, la pension était soustraite au salaire annuel de l'organiste titulaire. Le contrat de 1832 de Samuel Sebastian Wesley stipulait qu'il recevrait 40 livres sterling de plus par année à la mort de son prédécesseur, John Clarke-Whitfield (Massey et Shaw 2005, p. 15). En janvier 1843, c'est George Townshend Smith qui fut engagé à raison de 100 livres sterling moins les 25 livres sterling par année destinées à la veuve de son prédécesseur, John Hunt (ibid., p. 17).

\section{CONCLUSION}

La loi de l'offre et la demande peut être simplement définie comme « un mécanisme selon lequel l'accroissement de l'offre d'un produit quelconque sur le marché risque 
d'entraîner un fléchissement de son prix, alors qu'une demande accrue pour ce même bien risque, au contraire d'en faire monter le prix » (De Coster, Bawin-Legros et Poncelet 2006, p. 58). L'application du premier segment de cette théorie à la présente étude mène à la conclusion qu'un bassin plus important d'organistes - c'est-à-dire d'offreurs - entraîne une baisse de qualité des conditions de travail. De façon inversée, cette proposition signale que la rareté d'organistes permet d'obtenir de meilleures conditions de travail. Cette thèse est confirmée par l'étude de l'engagement des organistes de la cathédrale Holy Trinity entre 1801 et 1967, car les organistes du XIX ${ }^{e}$ siècle, qui étaient en situation de faible concurrence, ont bénéficié de façon quasi systématique des incitatifs à l'embauche (dont l'indemnité de déplacement et la hausse salariale à l'engagement) alors que ceux employés au $\mathrm{Xx}^{\mathrm{e}}$ siècle, qui faisaient face à une concurrence beaucoup plus importante, ont rarement eu droit à de tels incitatifs.

De 1801 à 1967, le rapport entre l'offre de service des organistes et la demande d'engagement d'organistes à la cathédrale Holy Trinity n'a pas toujours été équilibré. Dans la présente étude, le facteur temporel relatif à la durée de recherche d'un organiste est un important indicateur de décalage ou d'adéquation entre l'offre et la demande.

Alors que le poste d'organiste était habituellement comblé à l'intérieur d'un délai d'un à trois mois, il s'est écoulé, en 1874, six mois entre la démission de Henry Bradley et l'engagement d'Edward Arthur Bishop, ce qui dénote des difficultés de recrutement, et ainsi un décalage notable entre l'offre et la demande. De fait, durant ces six mois, deux organistes ont successivement été engagés, mais semblent s'être rétractés avant même d'avoir commencé leur mandat ${ }^{25}$. En plus, deux autres organistes qui avaient été sollicités personnellement, soit une mademoiselle McConnell ainsi que l'organiste de St. Bride de Liverpool, ont refusé le poste (CAQ, PV CF 26 mars et 14 avril 1874). Un dénommé Chamber avait également envoyé une lettre d'intention, mais le conseil de fabrique a préféré écarter cette candidature (CAQ, PV CF 26 mars 1874).

Le conseil de fabrique a connu de nouvelles difficultés de recrutement huit ans plus tard, à la suite de la démission de Bishop (CAQ, PV CF 22 décembre 1882). Même si deux organistes ont soumis leur candidature, soit C. A. Harriss d'Ottawa et un dénommé Jenny de Philadelphie (CAQ, PV CF 23 février 1883), aucun ne semblait satisfaire entièrement les dirigeants de la cathédrale. N'ayant pas d'autres candidats en vue, le recteur a donc urgemment demandé à Bishop de demeurer en poste et il a accepté (CAQ, PV CF 22 mars 1883).

De 1801 à 1967, l'étape de l'engagement a rarement été marquée par le "tâtonnement walrassien ", qui peut être défini comme "l'ajustement successif du prix et des quantités offertes et demandées " (Montoussé et Waquet 2008, p. 13). En fait, l'épisode de 1874 reste un évènement isolé. Cette année-là, le conseil de fabrique avait proposé un salaire de 600 dollars au dénommé Mills. Ce dernier a exigé

25 Le conseil de fabrique a approuvé l'engagement d'un dénommé Dickinson le 26 mars 1874 (CAQ, PV CF 26 mars 1874), puis celui d'un certain Mills le mois suivant (CAQ, PV CF 25 avril 1874). Ce « Mr. Mills » pourrait être Frederick William Mills, qui avait été organiste à la cathédrale Holy Trinity de 1865 à 1872 et qui a quitté Québec pour devenir organiste à l'église Christ Church d'Ottawa. 
un salaire plus élevé et le conseil a accepté. En concordance avec cette situation, les dirigeants de la cathédrale auraient pu négocier à nouveau les conditions de travail de Penn Spicer en 1932, dont 1'engagement à Holy Trinity était remis en question suite à une contre-offre de son ancien employeur, mais aucune démarche n'a été menée en ce sens. De façon générale, la demande - incarnée par les conditions de travail proposées par l'employeur - restait fixe et c'est l'offre, représentée par les exigences des organistes, qui s'ajustait en vue de trouver un " prix d'équilibre ", c'est-à-dire une entente d'engagement acceptée par les deux partis. En n'exigeant pas de conditions particulières lors de l'engagement, les organistes créaient ainsi une parfaite adéquation entre l'offre et la demande.

Ce rapport d'équivalence entre l'offre et la demande venait toutefois à être perturbé lors de la démission des organistes. L'arrêt de travail délibéré traduisait en fait un décalage entre l'offre et la demande. Cette perturbation était souvent créée par un facteur de demande concurrentielle, alors que d'autres églises, qui offraient des postes encore plus prestigieux ou des conditions de travail plus avantageuses, avaient besoin d'organistes. C'est pourquoi des organistes de Holy Trinity quittèrent leur emploi pour occuper des postes plus importants : John Carter à la cathédrale St. James de Toronto, Frederick William Mills à la future cathédrale Christ Church d'Ottawa, Maitland Farmer à l'église St. Paul de Toronto et Arnold Bellis fut éventuellement engagé à l'église unie St. James de Montréal. Ainsi, le poste à Holy Trinity a souvent servi aux organistes anglais de tremplin pour leur carrière nord-américaine. Pour les Anglais William Rains et Stephen Crisp, leur déménagement à Québec leur donna l'occasion d'accéder à un poste d'organiste titulaire dans une cathédrale, alors qu'ils étaient organistes assistants dans une cathédrale d'Angleterre.

Dans le but de conserver un équilibre entre l'offre et la demande durant le dernier quart du XIX ${ }^{\mathrm{e}}$ siècle, la communauté de la cathédrale de Québec a mis en place un système de rétention d'organiste basé sur la valorisation et l'implication. En fait, Arthur Edward Bishop avait reçu en 1877 des offres flatteuses et répétées des États-Unis, mais il avait ultimement décidé de les refuser, croyant que la congrégation de Holy Trinity l'encouragerait et coopèrerait avec lui dans ses efforts d'amélioration du chœur (CAQ, Annual Report of the Church Wardens of the Cathedral 1877, p. 9). Par conséquent, des remerciements lui furent adressés l'année suivante lors de l'assemblée des paroissiens (CAQ, Annual Report of the Church Wardens of the Cathedral 1878, p. 10). C'était la première fois qu'un organiste titulaire était publiquement remercié, alors que des félicitations annuelles systématiques au chœur bénévole étaient formulées depuis $1845^{26}$.

Cette valorisation ne fut probablement pas assez constante, car Bishop remit sa démission cinq ans plus tard, soit en décembre 1882. Toutefois, grâce aux efforts soutenus du recteur et de certains membres de la congrégation, Bishop a annoncé l'annulation de sa démission en mars 1883, en espérant « recevoir un meilleur soutien

26 La chorale bénévole de la cathédrale, qui remplaça le chœur de garçons et d'hommes vêtus de surplis, fut fondée durant la saison 1844-1845. 
de la part des paroissiens ${ }^{27}$ " (CAQ, PV CF 22 mars 1883). Bishop est finalement resté en poste jusqu'en 1924 grâce, entre autres, aux encouragements et à l'implication des choristes. En 1890, les marguilliers exprimaient d'ailleurs leur gratitude envers Bishop qui avait " réussi à positionner la chorale parmi les meilleurs chœurs des églises anglicanes du Canada ${ }^{28}$ " (CAQ, Annual Report of the Church Wardens of the Cathedral 1890, p. 11).

En somme, le rapport entre l'offre et la demande d'organistes à la cathédrale Holy Trinity fut généralement équilibré lors de l'engagement, mais le clergé, les marguilliers, le conseil de fabrique et les paroissiens ont dû développer différentes stratégies de rétention - notamment la valorisation des talents de l'organiste, l'implication des paroissiens dans la chorale bénévole, les hausses salariales pendant la période d'emploi et l'entretien constant de l'orgue - pour éviter un décalage entre l'offre et la demande, résultant habituellement par la démission ou la sollicitation d'une pension.

\section{LISTE DES ABRÉVIATIONS}

AS Assemblée des paroissiens

CAQ Fonds Cathédrale anglicane de Québec

$\mathrm{CF} \quad$ Conseil de fabrique

ESM Fonds Église St. Matthew, Québec

ESP Fonds Église St. Peter, Limoilou

ETC Fonds Église Trinity Church, Québec

PV Procès-verbal

\section{BIBLIOGRAPHIE}

Sources primaires

Fonds Cathédrale anglicane de Québec (Archives du diocèse anglican de Québec).

Fonds Église St. Matthew, Québec (Archives du diocèse anglican de Québec).

Fonds Église St. Peter, Limoilou (Archives du diocèse anglican de Québec)

Fonds Église Trinity Church, Québec (Archives du diocèse anglican de Québec).

\section{Sources secondaires}

Blakeley, Shirley A. (1993), "Farmer, Maitland », dans Helmut Kallmann et Gilles Potvin (dir.), Encyclopédie de la musique au Canada, 2e édition, vol. 1, Montréal, Fides, p. 1133.

27 "[...] in the hope of receiving greater support from the congregation".

28 " [...] has placed the Choir of the Cathedral of Quebec in the very front rank among choirs in connection with Anglican Churches through the Dominium ". 
Brouillette, Louis (2009), Le "Manuscrit d'orgue de la cathédrale anglicane de Québec ». Description, analyse et mise en contexte, Thèse de doctorat, Université de Montréal.

Cahill, Barry et al. (2008), The Blue Banner. The Presbyterian Church of Saint David and Presbyterian Witness in Halifax, Montréal, McGill - Queen's University Press.

De Coster, Michel, Bawin-Legros, Bernadette, et Marc Poncelet (2006), Introduction à la sociologie, $6^{\mathrm{e}}$ édition, Bruxelles, De Boeck.

Defalvard, Hervé (2003), Fondements de la microéconomie, «Vol. 2. L'équilibre des marchés », préface de Roger Guesnerie, Bruxelles, De Boeck.

Émond, Vivianne (1986), «"Musique et musiciens à Québec. Souvenirs d'un amateur" de Nazaire Levasseur (1848-1927). Étude critique », Mémoire de maîtrise, Université Laval.

Hayes, Florence, McGregor, Nancy, et Jeanne Rizzo (1993a), « Carter, Henry », dans Helmut Kallmann et Gilles Potvin (dir.), Encyclopédie de la musique au Canada, $2^{\mathrm{e}}$ édition, vol. 1, Montréal, Fides, p. 523.

Hayes, Florence, McGregor, Nancy, et Jeanne Rizzo (1993b), « Carter, John », dans Helmut Kallmann et Gilles Potvin (dir.), Encyclopédie de la musique au Canada, $2^{\mathrm{e}}$ édition, vol. 1, Montréal, Fides, p. 523.

Lefebvre, Marie-Thérèse, et Jean-Pierre Pinson (2009), Chronologie musicale du Québec, 1535-2004. Musique de concert et musique religieuse, avec la collaboration de Mireille Barrière et al., Québec, Septentrion.

Massey, Roy, et Watkins Shaw (2005), The Organists and Organ of Hereford Cathedral, Hereford, Hereford Cathedral Organ Committee.

Montoussé, Marc, et Isabelle Waquet (2008), Microéconomie. Introduction à l'économie, $2^{\mathrm{e}}$ édition, Rosnysous-Bois, Bréal.

Poirier, Lucien (1993), « Codman, Stephan », dans Helmut Kallmann et Gilles Potvin (dir.), Encyclopédie de la musique au Canada, $2^{\mathrm{e}}$ édition, vol. 1, Montréal, Fides, p. 672-673.

Ryder, Dorothy (1983), « Bentley, John », dans Francess G. Halpenny (dir.), Dictionnaire biographique du Canada, «Vol. 5. 1801-1820 », Québec, Presses de l'Université Laval, p. 73-75.

Ryder, Dorothy (1993), «Bentley, John », dans Helmut Kallmann et Gilles Potvin (dir.), Encyclopédie de la musique au Canada, 2 édition, vol. 1, Montréal, Fides, p. 278.

Waterlow, Barry D. (1993), "Chubb, Frederick », dans Helmut Kallmann et Gilles Potvin (dir.), Encyclopédie de la musique au Canada, $2^{\mathrm{e}}$ édition, vol. 1, Montréal, Fides, p. 642. 